\title{
Blind Standard Identification with Bandwidth Shape and GI Recognition using USRP Platforms and SDR4all Tools
}

\author{
H. Wang, W. Jouini, A. Nafkha, and J. Palicot \\ SCEE/IETR \\ SUPELEC,Avenue de la Boulaie,CS 47601 \\ 35576 Cesson Sévigné Cedex, France \\ Email: \{hongzhi.wang, wassim.jouini, \\ amor.nafkha, jacques.palicot\}@ supelec.fr
}

\author{
L. S. Cardoso and M. Debbah \\ Alcatel-Lucent Chair \\ SUPELEC, 3 rue Joliot-Curie, 91192 CEDEX \\ Gif-sur-Yvette, France \\ Email: \{leonardo.cardoso, merouane.debbah\}@ supelec.fr
}

\begin{abstract}
In this paper, focusing on identifying standards blindly, we propose a bandwidth shape sensor and a GI (guard interval) sensor using USRP (Universal Software Radio Peripheral) platforms and SDR4all tools. These sensors are fundamental parts of the so-called Blind Standard Recognition Sensor. The blind standard bandwidth sensor is based on a Radial Basis Function Neuronal Network designed in Matlab. We have presented first experience of using blind standard bandwidth sensor in a previous work. We will provide in this paper further details on the results of this sensor (simulations, preliminary implementations and validations). The GI sensor is implemented in order to improve the detection performance in the case of two identical bandwidth shapes. The SDR4all driver offers a simple yet efficient interface between the Matlab signal processing codes and the USRP transmitting and receiving platforms. These simple and easily accessible software defined radio tools were used to design and implement two sensors. The inducted simulations and experiments show that the designed system is indeed able to discriminate three standard-like spectrums (e.g., GSM-like, UMTS-like and OFDM-like) under simple yet real transmission conditions using their different bandwidth shapes and to identify a GI-OFDM-like system using cyclic autocorrelation method.
\end{abstract}

Keywords-Cognitive Radio; Blind Standard Identification Sensor, Sensorial Radio Bubble, SDR4all, USRP.

\section{INTRODUCTION}

Cognitive Radio (CR) presents itself as a set of concepts and technologies that enable radio equipments to have the autonomy and the cognitive abilities to become aware of their environment as well as of their own operational abilities [1]. Thus it is a device that has the ability to collect information through its sensors and that can use the past observations on its surrounding environment to improve its behavior accordingly. In order to make appropriate decisions, cognitive radio equipments rely on the gathered information from the sensors. Consequently, designing simple, accurate and reliable sensors enable the CR equipment to adapt itself in the best possible way to its surrounding environment. In [2], the concept of "Sensorial Radio Bubble" (SRB) has been introduce to model and process the environment seen from the several sensors of the equipment. Thus it can be seen as the smallest cluster associated to the $\mathrm{CR}$ equipment. Through this cluster, the $\mathrm{SRB}$ has to manage (discriminate, fusion, filter, etc.) raw information collected from its different sensors in order to send reliable and useful information to the decision making engine inside the equipment.

Within the SRB context, one of the most important sensors is the "Blind Standard Recognition Sensor" (BSRS) [3]. Blind refers to the capabilities of the system to identify a standard based on the characteristics of the received signal. These characteristics are limited to the band shape and possibly other metrics (e.g., single/multicarrier signal, frequency hopping or direct sequence access, etc.) to enable discriminating standards with similar band shapes. It is true that today the standards have known transmission frequencies, however within a cognitive radio context, this constraint is alleviated. Therefore, since the parameters are inferred from the environment and no more dictated by the network, several steps are needed and "blindly" conducted to identify a standard within the frequency range of a cognitive radio receiver. Thus, the BSRB is composed of three steps: band adaptation, metric analysis (i.e., extraction of different features from the received signal) and information fusion step (Figure 1).

In this paper, we implement the blind standard bandwidth shape sensor of the BSRS to discriminate standard-like signals as well as a Guard Interval (GI) sensor using Matlab, SDR4all tools and USRP platforms. The metric analysis step for bandwidth sensor is based on a Radial Basis Function Neuronal Network (RBF-NN) described in Section II as well as cyclic autocorrelation method for GI sensor. All signal processing blocks (transmission of the standard-like signals, bandwidth adaptation, feature analysis, etc.) are coded in Matlab while SDR4all tools as well as the USRP platforms deal with the transmission and reception of the waveforms (Section III). Simulation results of detection performances will be provided in section IV. The experimental results given in section $\mathrm{V}$ shows that we can indeed discriminate these signals after a transmission through a simple yet real channel. 


\section{BLIND STANDARD IDENTIFICATION FOR COGNITIVE RADIO RECEIVERS}

The SRB relies on a multitude of sensors based on several signal processing blocks. It gives communication systems the ability to explore the radio environment in order to gain knowledge on the spatial and spectrum environment while providing the system with some context awareness. The sensors of the SRB are fully described in [2].

The BSRS presented in [3] is a fundamental example among the set of SRB sensors for cognitive radio equipments. This sensor aims at identifying all possible standards in the vicinity of the received area. It can also be used to estimate the spectrum occupancy [4]. For that purpose, the standard uses a set of information about the received signals such as the bandwidth, spreading techniques or cyclostationarity features. We focus on two parameters: the bandwidth shape recognition performed by a neuronal network and cyclostationarity techniques to detect the GI.

\section{A. The bandwidth recognition}

The bandwidth shape recognition is used to identify blindly the standards of the received signals in the paper [5]. The author claimed that this parameter was enough to discriminate all commercialized wireless standards, which was true in 2003, however with the growing number of new standards introduced on the market it is not true anymore and a full BSRS is needed (cf. Figure 1 and paper [2]).

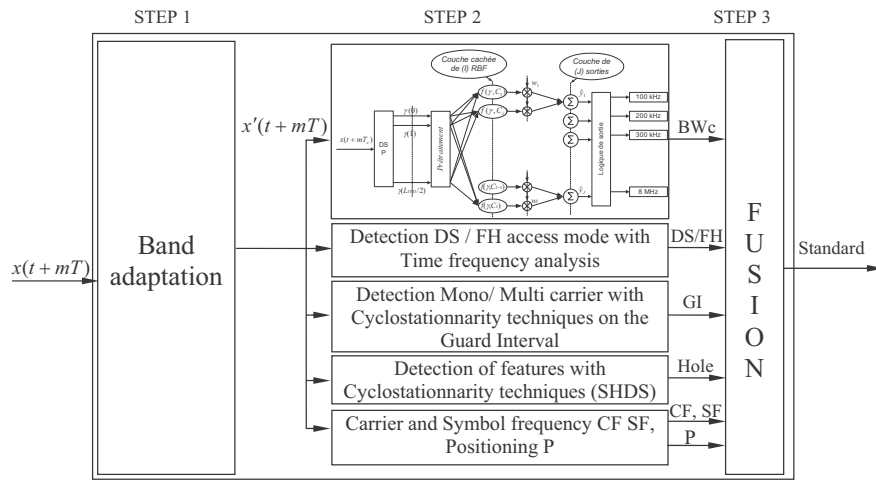

Fig. 1. The Blind Standard Recognition Sensor.

In this paper, we use the bandwidth shape parameters to identify blindly a set of standard-like signals. For the sake of simplicity we constrain our experiments to three types of signals: GSM-like, UMTS-like, WiFi-OFDM-like (Section V). We use, interchangeably, the notations "GSM/GSM-like", "UMTS/UMTS-like" and "OFDM/OFDM-like", since we do not respect the bandwidths of these standards for practical reasons. In this situation the received signal's bandwidth shape is a criterion that fully discriminates the three standards. Examples of real bandwidth shape parameters associated to several standards are given in Table I.

We consider a set of signals that can be fully discriminated using the bandwidth and filter shape parameters. We know that a large class of standards is included in this set. To find the
TABLE I

BANDWIDTH PARAMETERS USED TO DISCRIMINATE DIFFERENT STANDARDS.

\begin{tabular}{|c|c|c|}
\hline standards & channel bandwidth & shape filter \\
\hline PDC & $25 \mathrm{kHz}$ & SRC $(\alpha=0.5)$ \\
\hline ADC (D-AMPS) & $30 \mathrm{kHz}$ & SRC $(\alpha=0.5)$ \\
\hline CT2 & $100 \mathrm{kHz}$ & Gaussian 0.5 \\
\hline GSM & $200 \mathrm{kHz}$ & Gaussian 0.3 \\
\hline Bluetooth & $1 \mathrm{MHz}$ & Gaussian 0.5 \\
\hline DAB & $1.712 \mathrm{MHz}$ & Window \\
\hline DECT & $1.728 \mathrm{MHz}$ & Gaussian 0.5 \\
\hline UMTS (FDD) & $5 \mathrm{MHz}$ & SRC $(\alpha=0.2)$ \\
\hline DVB-T, LMDS & $7-8 \mathrm{MHz}$ & Window \\
\hline Hiperlan I & $20 \mathrm{MHz}$ & Gaussian 0.5 \\
\hline DVB-S & $32-36 \mathrm{MHz}$ & SRC $(\alpha=0.3)$ \\
\hline Hiperlan II & $50 \mathrm{MHz}$ & Window \\
\hline
\end{tabular}

bandwidth shape on the received signal a choice has been made to perform a power spectrum density (PSD) on this signal in order to obtain the shape of its bandwidth. This shape is compared with reference bandwidth shapes considered as the database of the sensor used to identify the standards. The mathematical expression of these features is given by Equation (1).

$$
\gamma_{r e f}(k)=\left|\operatorname{Fem}_{s}\left(\frac{f_{p}}{f_{e}}-k\right)\right|^{2} \gamma_{\bmod }\left(\frac{f_{p}}{f_{e}}-k\right)
$$

where $\gamma_{r e f}$ is the PSD of a reference signal and $\gamma_{\text {mod }}\left(\frac{f_{p}}{f_{e}}-k\right)$ is the PSD associated to the considered modulation.

Finally, we aim at comparing the shape of the received signal to the reference standards' shapes. The design of the sensor is based on a RBF-NN (Radia Basis Functional Neural Networks) that is known to be efficient when dealing with pattern recognition related problems. Several NN classes are described in the literature [6] in which the reader can find all the basic information.

In this work, we determine the following expression to maximize the distance between the reference signal $\gamma_{r e f}$ and the received signal $\gamma$.

$$
M S E=\frac{1}{L_{i}} \sum_{l=1}^{L_{i}}\left(\left(\gamma(l)-\gamma_{r e f, i}(l)\right)^{2}\right)
$$

where $L_{i}$ refers to the number of reference standards in the database considered to compute the error function.

Thus, as mentioned before, a power spectrum density (PSD) is performed on the received signal. Then using the RBFNN with the error function defined by (2), the PSD of the received signal is compared to ones present in the reference database. In order to determine the RBF-NN's thresholds, we built a confusion matrix that determines the cross-error between the different references. It can be interpreted as the distance between the considered patterns. The larger the crosserror between two references features is, the easier it will be to separate them.

\section{B. Cyclostationarity detection on Guard Interval}

As the analyzed signal is a telecommunication signal, an interesting alternative consists in choosing a cyclostationarity [7] 
model instead of a stationary model of the signal. This model is all the more interesting under the assumption of a stationary noise. As a matter of fact, the detection problem becomes equivalent to test the signal's cyclostationarity characteristics. A process $x(t)$ is said to have a cyclostationarity property in a wide sense if its mean, $m_{x}$, and cyclic autocorrelation, $R_{x}$, are periodic with a period of $T: m_{x}(t+T)=m_{x}(T) . R_{x}$ is a function of two independent variables $t$ and $\tau$ such that, it is periodic with respect to the variable $t$ with period $T$ for every value of $\tau: R_{x}(t, \tau)=R_{x}(t+T, \tau)$. Consequently, we can write using Fourier series with variable $t$ :

$$
R_{x}(\alpha, \tau)=\frac{1}{T} \int_{-T / 2}^{T / 2} R_{x}(t+T, \tau) e^{-i 2 \pi \alpha t} d t
$$

Equation 3 is made of harmonics of the fundamental frequencies, determined by the periods of $R_{x}(t, \tau)$. These fundamental frequencies either represent carrier frequencies, data rate frequencies, or guard intervals of the signal, etc. Using the previously introduced notations, the parameter $\alpha$ is referred to as cyclic frequency.

We investigate the implementation of cyclostationarity detection to improve detection performance by identifying OFDM symbol's GI in case of two identical bandwidth shapes for GI-OFDM and GI-less OFDM systems. An implementation of the designed system is then realized using SDR4all tools and USRP platforms. Cyclostationarity methods are known to be efficient. Unfortunately, their complexity makes their computation, in some contexts, burdensome. Nevertheless, within the herein described context, the analysis of the GI can be simplified and a simple autocorrelation is sufficient.

\section{SDR EVALUATION TOOL}

The evaluation of the proposed algorithm was done with a testbed called SDR4all. It is composed of a USB plug and play hardware part, and a software part. The hardware part is in charge of the RF and sampling processing while the radio transceiver's physical layer (PHY) is software driven, running in a computer.

\section{A. Hardware}

In this evaluation, the hardware of the testbed was based on the USRP version 1 cards [8]. However, SDR4all can be used on other types of radio cards (such as the ones being developed by SDR4all [9]). These cards are divided into two parts: a mother-board and one or two daughter-boards. The motherboard is responsible for the RF control, communication over the USB link, analog-to-digital/digital-to-analog conversions and sampling. The daughter-board is responsible for the RF circuitry, including filters, amplifiers and oscillators. Note that the USRP version 1 accepts a wide range of daughter-boards, available to allow testing in multiple bands [8].

As of now, SDR4all supports the RF circuitry provided by the RFX2400 daughter-board. The $2.4 \mathrm{GHz}$ ISM band was chosen since it shares the same characteristics with the widely popular 802.11(b/g), bluetooth and WiMAX systems. Along with the RFX2400 daughterboard, dual VERT2450 antennas
(TX and RX, each) are adopted. These are standard isotropic antennas made for the 2.4 and $5 \mathrm{GHz}$ ISM band. The main parameters for the mother-board and RF circuitry are provided in table II [8].

\begin{tabular}{c|c}
\hline parameter & value \\
\hline operating band & ISM $2.4 \sim 2.49 \mathrm{GHz}$ \\
base-band filtering & $20 \mathrm{MHz}$ \\
channels & 1 to $13(802.11)$ \\
total TX power & $20 \mathrm{~mW}$ \\
signal bandwidth & up to $16 \mathrm{MHz}$ \\
\hline
\end{tabular}

TABLE II

PARAMETERS FOR THE HARDWARE PART.

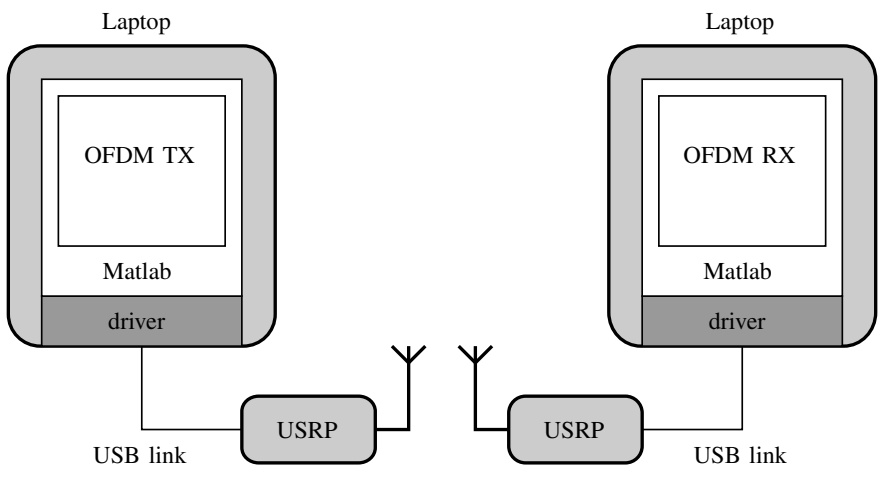

Fig. 2. SDR4all overall layout

\section{B. Software}

As previously stated, the PHY layer is implemented in software, part of a Matlab toolbox developed specifically to this end. In order for the toolbox to correctly communicate with the cards, a driver was built. At the moment, the driver enables non real-time communication between only a card and a computer, but multi-card, real-time operation is currently under development. It is able to transmit a given vector of baseband I/Q symbols and to listen for a certain amount of time and provide a vector of the received baseband I/Q samples. The driver also allows to configure the bandwidth and the center frequency for both transmission and reception. The toolbox implements basic communication blocks, such as bit operations, modulation, packet formatting and so on. Full communication chains are also implemented. The overall schematics of SDR4all, for an example OFDM chain, can be seen in Figure 2.

Next section describes the experimental protocol and presents the main results of the implemented system using, Matlab, SDR4all tools and USRP platforms.

\section{Simulation RESUlts}

In this section, we provide simulation results demonstrating the performance of the proposed blind standard detection and identification method. Two simulations are carried out to evaluate the performance of the proposed bandwidth shape recognition and GI detection method. First we start by generating the source signals used for the recognition process. 


\begin{tabular}{c|c}
\hline parameter & value \\
\hline$f_{c}$ & flexible inside the ISM band \\
base band rate & $1 \mathrm{MHz}$ \\
$n_{\text {pre }}$ & $1000 \mathrm{syms}$ \\
$n_{F F T}$ & 64 \\
$n_{z \text { pad }}$ & 16 (8 at each end $)$ \\
$N$ & 48 carriers \\
$n_{\text {pilots }}$ & 30 \\
$n_{\text {data }}$ & 30 \\
pilot structure & QPSK or circularly symmetric \\
data modulation & 16-QAM \\
\hline
\end{tabular}

TABLE III

PARAMETERS FOR THE ALGORITHM MODE.

\section{A. References and database}

For the sake of simplicity we restrain our data base to three sources. In order to test our demonstration, we generated standard-like signals using Matlab. We refer to these sources as GSM, UMTS and WiFi-OFDM-like signals. Mostly because of hardware limitations (especially for WiFi-like signals), we limited the characteristics of the used signals to their filter shape. Thus the bandwidths are not respected. Moreover, we assume that the band adaptation step (c.f. Figure 1) was successful. However, it is sufficient to demonstrate how this sensor works. The characteristics of these sources are the following:

- GSM modulation is GMSK (Gaussian filtered MSK). GMSK is a MSK with a Gaussian Filter of an appropriate bandwidth.

- Universal Mobile Telephone System (UMTS) uses rootraised cosine (RRC) as the pulse-shaping filters. In UMTS system, the modulation used is the Quadrature Phase Shift Keying (QPSK).

- In an OFDM scheme, 64 subcarriers are employed in the source signal (c.f. Table III). The transmitter first converts the input data from a serial stream to parallel sets. And an inverse Fourier transform converts the frequency domain data set into samples of the corresponding time domain representation of this data.

These signals are then stored in a binary file and considered as the database of the receiver. As described in Section II, these sources are compared to the received signals through an RBF$\mathrm{NN}$ in order to identify the standards. In order to identify a standard, the reference spectrum are generated using the source file, as shown in the Figure 3.

\section{B. Bandwidth shape recognition performance}

The received signals are generated by adding the additive white Gaussian noise (AWGN) on the reference signals. Identification performance using the bandwith shape recognition is evaluated in term of probability of successful identification under different SNRs. The simulation results are shown in Figure 4.

In this simulation example, the result is evalutated on mean performance of three standards which are considered

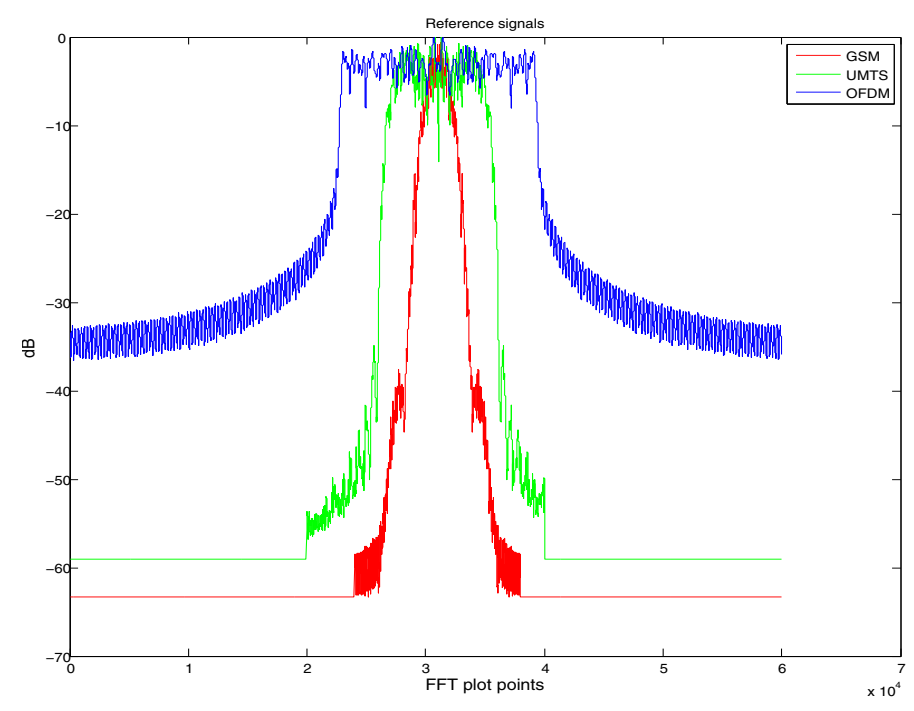

Fig. 3. Standard-like PSD patterns.

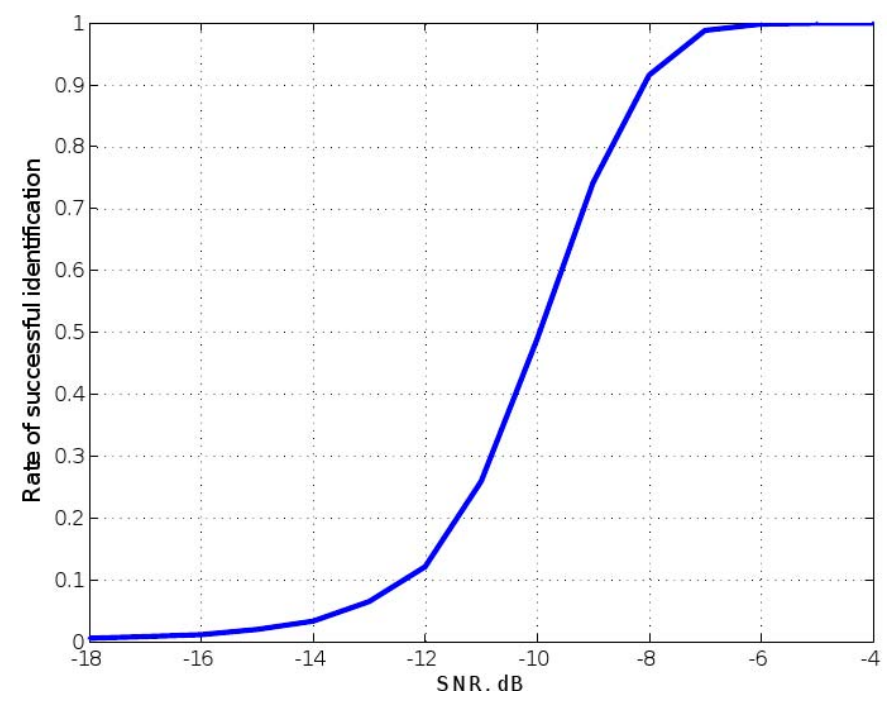

Fig. 4. Simulation results on detection performances. The curve takes into account the three standard-like signals analyzed.

as equiprobable. The bandwidth shape detection performance is obtained with a false alarm probability lower than $0.5 \%$.

\section{GI detection performance}

In this simulation example, we employ the simple peak detection method on the OFDM symbol with GI. In the paper [10], authors showed that peak detection is a good method with low false alarm probability. A large amount of subcarriers $2 \mathrm{k}$ with $1 / 4$ length of guard interval is used in order to achieve better detection performance. Figure 5 illustrates the performance of the peak detection as a function of the SNR. It shows that the peak detection based on the autocorrelation method is reliable to identify the GI in OFDM system. 


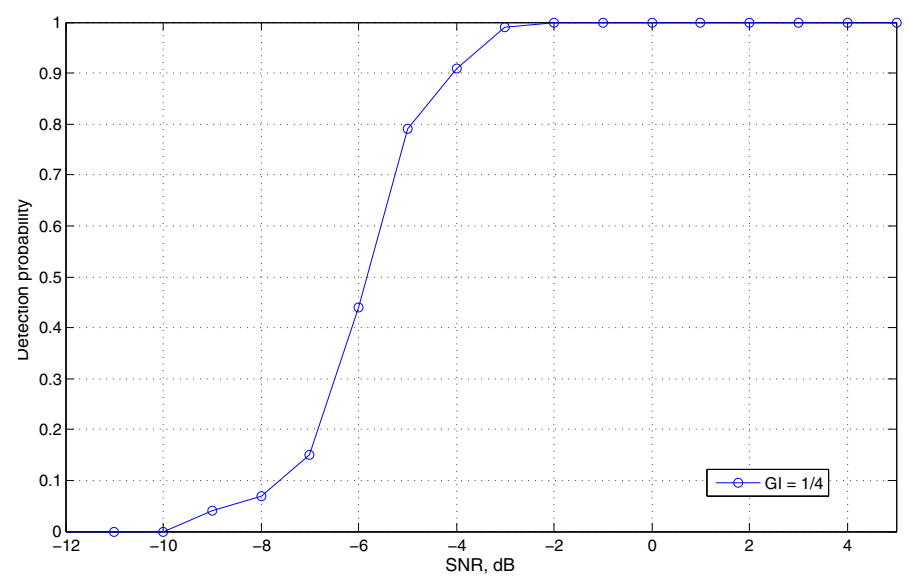

Fig. 5. Simulation results: GI-OFDM's detection probability depending on the SNR.

\section{EXPERIMENTAL RESULTS}

\section{A. Experimental protocol}

In this section we describe the experimental protocol used on the USRP platforms and present the main results.

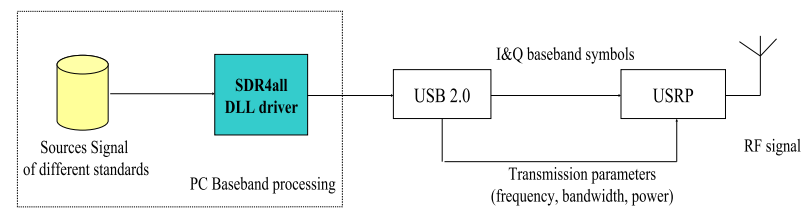

Fig. 6. Architecture of the transmitter

1) Transmitter: The transmitter is described in Figure 6. At the transmitter we can select the source to send. In order to continuously transmit (which is not possible using Matlab), we designed a $\mathrm{C}$ component that takes the binary source files and repeatedly send them as a vector of I/Q baseband symbols to the SDR4all driver presented in the Section III. This latter sends the stream to the USRP which transmits the data at a center frequency inside the ISM band.

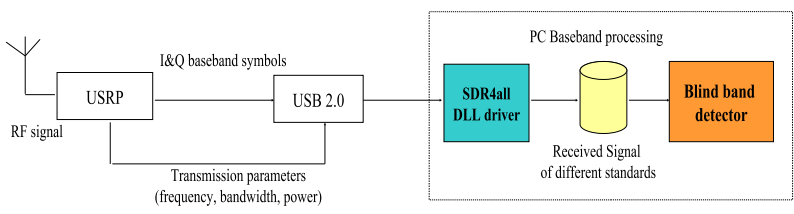

Fig. 7. Architecture of the receiver.

2) Receiver: Figure 7 shows the block diagram of the receiver. The SDR4all driver is also used to collect the data from the USRP platform over a USB link. The receiver chooses the same center frequency as the transmitter and takes a period of the transmitted signals in the form of I/Q baseband samples. Since Matlab cannot deal with the received signals in real-time, these samples are also stored into a binary file which is then read by the band detector implemented in Matlab in order to compute the signal's PSD. Finally, the PSD of the received signals are compared to the ones in the database (reference signals) in order to identify the standard. Similarly, the GI detector reads samples from the binary file and performs an autocorrelation function on the signal in order to identify GI OFDM system by detecting the peaks.

\section{B. Results}

A detection probability of $100 \%$ is confirmed for SNRs higher than $0 \mathrm{~dB}$. In order to present the real capabilities of the system, extensive tests are currently conducted on the USRP platforms. We aim at showing that the performances of the herein described sensors are comparable to those found in simulations (c.f. Figure 4 and 5). The results of the ongoing experiments will be presented in a future work taking into account a larger number of standards and sensors.

\section{CONCLUSION AND FURTHER WORK}

In this paper, we implemented a BSRS in order to discriminate standardized-like signals. The bandwidth sensor is based on a PSD shape comparison between references and the received. The comparison is performed by a RBF-NN and a GI sensor. The main purpose of this paper is to show the possibility to implement such systems using intuitive tools such as Matlab and SDR4all tools, and, open source hardware such as the USRP platforms. Although the design of a complete and real time operational BSRS is still in its infancy, we show in this paper that we can indeed blindly discriminate, at the receiver, signals with different shapes after a transmission through a real channel. Currently, experiments are conducted to quantify more precisely the performance of the designed system on real channels. The results of these ongoing experiments will be presented in our future works.

\section{ACKNOWLEDGMENT}

This work was supported by the European Commission in the framework of the FP7 Network of Excellence in Wireless COMmunications NEWCOM++ (contract n. 216715).

\section{REFERENCES}

[1] J. Mitola III and GQ Maguire Jr. Cognitive radio: making software radios more personal. IEEE personal communications, 6(4):13-18, 1999.

[2] J. Palicot, C. Moy, and R. Hachemani. Multilayer sensors for the sensorial radio bubble. Physical Communication, (2):151-165, May 2009.

[3] R. Hachemani, J. Palicot, and C. Moy. A new standard recognition sensor for cognitive terminal. In Proceeding in The European Signal Processing Conference, 2007.

[4] M. Lopez-Benitez, F. Casadevall, A. Umbert, J. Perez-Romero, R. Hachemani, J. Palicot, and C. Moy. Spectral occupation measurements and blind standard recognition sensor for cognitive radio networks. CrownCom'09, Hannover, Germany, June 2009.

[5] C. Roland and J. Palicot. A new concept of wireless reconfigurable receiver. IEEE communications Magazine, July 2003.

[6] A. Cichocki and R. Unbehauen. Neural networks for optimization and signal processing, wiley. 1993.

[7] W.A. Gardner. Statistical spectral analysis: A non probabilistic theory. Prentice Hall, Englewood Cliffs, NJ, 1988.

[8] http://www.ettus.com.

[9] http://www.sdr4all.org. 2008.

[10] Sung Hwan Sohn, Ning Han, Jae Moung Kim, and Jae Wan Kim. Ofdm signal sensing method based on cyclostationary detection. Cognitive Radio Oriented Wireless Networks and Communications, 2007. CrownCom 2007. 2nd International Conference on, pages 63 -68, aug. 2007. 